\title{
Prevention strategies for type 2 diabetes should be based on evidence-based medical nutrition data
}

\author{
M. Uusitupa $・$ J. Lindström $・$ J. Tuomilehto
}

Received: 11 March 2010 /Accepted: 1 April 2010 /Published online: 8 May 2010

(C) Springer-Verlag 2010

Keywords Diet $\cdot$ Nutrition $\cdot$ Prevention $\cdot$ Randomised controlled trial · Type 2 diabetes

\section{Abbreviations \\ DPP The Diabetes Prevention Program \\ DPS The Diabetes Prevention Study \\ E\% Percentage of energy}

To the Editor: In an extensive review Buyken and coauthors discussed the optimal dietary approaches for the prevention of type 2 diabetes [1]. The topic is of utmost importance because of the alarming increase in obesity, cardiometabolic syndrome and type 2 diabetes, the modern worldwide epidemic. The authors very briefly bypass the evidence coming from two lifestyle intervention trials, the

\section{Uusitupa}

Institute of Public Health and Clinical Nutrition,

University of Eastern Finland,

P.O. Box 1627, 70211 Kuopio, Finland

M. Uusitupa $(\bowtie)$

Food and Health Research Centre,

Kuopio, Finland

e-mail: matti.uusitupa@uef.fi

\section{J. Lindström}

Diabetes Prevention Unit, Department of Chronic Disease

Prevention, National Institute for Health and Welfare,

Helsinki, Finland

J. Tuomilehto

Hjelt Institute, Department of Public Health,

University of Helsinki,

Helsinki, Finland

J. Tuomilehto

South Ostrobothnia Central Hospital,

Seinäjoki, Finland
Diabetes Prevention Study (DPS) [2] from Finland and the Diabetes Prevention Program (DPP) [3] from the USA, which showed a marked reduction in the incidence of diabetes both during the active intervention and the subsequent follow-up of up to 10 years $[4,5]$. The authors then extensively discuss alternative diets that supposedly might be helpful in the prevention of type 2 diabetes. However, two major concerns remain after having read this review.

First, the authors do not give major emphasis to evidence-based medical nutrition. In this regard, randomised controlled trials with onset of diabetes as an endpoint give the strongest evidence. But we do not yet have a definite answer to the question 'what kind of diet is the best to prevent type 2 diabetes', or even if such a universally superior diet exists. What we do know is that weight reduction and physical activity are the cornerstones of prevention. Weight reduction, however, is only possible with the reduction of total energy intake by dietary changes or increasing physical activity. As for the hard endpoint, the above-mentioned randomised prevention trials give the best (class A) evidence we have at the moment evidence and deserve to be cited appropriately.

As for the quality of diet, we in the DPS study group originally decided to follow the general dietary recommendations for Nordic countries, with minor modifications. Information on the diets recommended and achieved in the DPS, as well as on how the goals were translated to practical dietary advice has been presented in detail [6]. The goal of total fat intake was $30 \%$ of energy ( $\mathrm{E} \%$; intake up to $35 \mathrm{E} \%$ was acceptable if the surplus was monounsaturated fat), which can be considered a 'moderate' rather than a 'low' fat intake. Baseline total fat intake was 36-37 E\% and intake of saturated fat $16-17 \mathrm{E} \%$. After 1 and 3 years of the active intervention there was a $3.4-4.7 \mathrm{E} \%$ reduction in 
total fat and a 2.7-3.2 E\% reduction in saturated fat in the intervention group. Carbohydrate intake was $43 \mathrm{E} \%$ at the start and rose by $3.3 \mathrm{E} \%$, with no difference between the years 1 and 3 . However, the total intake of carbohydrates as measured in $g$ was actually slightly reduced at 1 and 3 years, respectively, because of a lower total energy intake [8]. Simultaneously, the intake of dietary fibre was increased in the intervention group, in line with the recommendation. Therefore, labelling the diet recommended in the DPS 'a conventional low-fat high-carbohydrate diet' [1] is a misleading. Of note, the weight reduction in both the DPS and the DPP was comparable with that in the studies with different diet modifications cited by Buyken et al. [1]. Importantly, in the DPS the incidence of diabetes was lowest (almost complete protection) and weight reduction greatest among people who were able to achieve four or five of the lifestyle intervention goals (weight loss $>5 \%$, intake of fat $<30 \mathrm{E} \%$, intake of saturated fats $<10 \mathrm{E} \%$, intake of dietary fibre $\geq 15 \mathrm{~g} / 4,200 \mathrm{~kJ}[1,000 \mathrm{kcal}]$ and physical activity for at least $4 \mathrm{~h} /$ week) $[2,5]$. This provides clear evidence in support of the chosen intervention strategy.

Second, the authors do not discuss at all the quality of dietary fat, which is of particular importance for the prevention of cardiovascular disease. In a recent meta-analysis on the effect of reducing saturated fat on mortality and morbidity from cardiovascular disease no significant association was found [7]. The paper has attracted great attention from both the medical community and the popular media. However, the study is open to serious criticism, such as that regarding the adjustment for serum cholesterol in the analyses [8]. This, of course, is a problem as the effect of saturated fat on cardiovascular disease is believed to be mediated mainly through serum lipids. Furthermore, the meta-analysis does not take into account the trial evidence available.

Saturated fat should be replaced by unsaturated fat not only because of serum lipids but also because of other benefits such as, for example, for insulin sensitivity [9]. In the DPS, we also found beneficial changes in inflammatory markers [10], and blood pressure [11]. These also suggest that a moderate relative increase in carbohydrates, e.g. increasing intake of wholegrain food products, while keeping fat intake to a moderate level and emphasising adequate intake of unsaturated fat makes sense. Weight reduction was not the only factor that explained these changes and, particularly, the increase in the intake of dietary fibre was positively related to the decrease in inflammatory factors [10]. Compared with the Finnish population with normal glucose tolerance, the participants in the DPS, who all had impaired glucose tolerance at baseline, had a significantly lower mortality, by about $60 \%$, during the 10-year follow-up [12].
We do agree that the dietary recommendations for the prevention and dietary treatment of type 2 diabetes may need to be sharpened: for example, in terms of carbohydrate sources. Also, the quality of fats should be taken seriously. In general, we should focus on diet as a whole, on foods and dietary patterns rather than on nutrients, and understand that changing something in the diet will affect other factors as well. In that framework, the diet recommended in the DPS and of proven effectiveness remains valid. All recommended changes should be based on well-documented scientific data. In this regard, randomised controlled long-term trials give the strongest evidence so far.

Duality of interest The authors declare that there is no duality of interest associated with this manuscript.

\section{References}

1. Buyken AE, Mitchell P, Ceriello A, Brand-Miller J (2010) Optimal dietary approaches for prevention of type 2 diabetes: a life-course perspective. Diabetologia 53:406-418

2. Tuomilehto J, Lindström J, Eriksson JG et al (2001) Prevention of type 2 diabetes mellitus by changes in lifestyle among subjects with impaired glucose tolerance. N Engl J Med 344:1343-1350

3. The Diabetes Prevention Program Research Group (2002) Reduction in the incidence of type 2 diabetes with lifestyle intervention or metformin. N Engl J Med 346:393-403

4. Lindström J, Ilanne-Parikka P, Peltonen M et al (2006) Sustained reduction in the incidence of type 2 diabetes by lifestyle intervention: the follow-up results of the Finnish Diabetes Prevention Study. Lancet 368:1673-1679

5. Knowler WC, Fowler SE, Hamman RF et al (2009) 10-year follow-up of diabetes incidence and weight loss in the Diabetes Prevention Program Outcomes Study. Lancet 374:1677-1686

6. Lindström J, Louheranta A, Mannelin M et al (2003) The Finnish Diabetes Prevention Study (DPS): Lifestyle intervention and 3 -year results on diet and physical activity. Diab Care 26:3230 3236

7. Siri-Tarino PW, Sun Q, Hu FB, Krauss RM (2010) Meta-analysis of prospective cohort studies evaluating the association of saturated fat with cardiovascular disease. Am J Clin Nutr 91:535-546

8. Stamler J (2010) Diet-heart: a problematic revisit. Am J Clin Nutr 91:497-499

9. Vessby B, Uusitupa M, Hermansen K et al (2001) Substituting dietary saturated for monounsaturated fat impairs insulin sensitivity in healthy men and women: the KANWU Study. Diabetologia 44:312-319

10. Herder C, Peltonen M, Koenig W et al (2009) Anti-inflammatory effect of lifestyle changes in the Finnish Diabetes Prevention Study. Diabetologia 52:433-442

11. Ilanne-Parikka P, Eriksson JG, Lindström J et al (2008) Effect of lifestyle intervention on the occurrence of metabolic syndrome and its components in the Finnish Diabetes Prevention Study. Diab Care 31:805-807

12. Uusitupa M, Peltonen M, Lindström J et al (2009) Ten-year mortality and cardiovascular morbidity in the Finnish Diabetes Prevention Study - secondary analysis of the randomized trial. PLoS ONE 4:e5656 